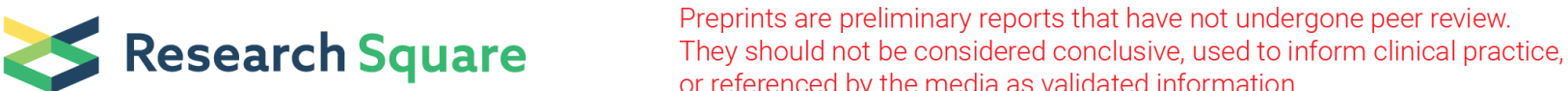

\section{Platelet-To-Lymphocyte Ratio Can Be Used As Marker To Predict Activity And Severity In Moderate And High Disease Activity of Rheumatoid Arthritis: A Retrospective Study}

\section{Bilin Chen}

Central South University

Qing Zhu

Central South University

Shu Li

Central South University

Yan Ge

Central South University

Peijun Wu

Central South University

Jing Tian

Central South University

Xi Xie

Central South University

Fen Li ( $\square$ lifen0731@csu.edu.cn )

Central South University

Research Article

Keywords: rheumatoid arthritis, platelet / lymphocyte ratio, neutrophil / lymphocyte ratio, disease activity score, grey scale ultrasound score, power doppler ultrasound score

Posted Date: December 20th, 2021

DOI: https://doi.org/10.21203/rs.3.rs-1077543/v2

License: @ (1) This work is licensed under a Creative Commons Attribution 4.0 International License. Read Full License 


\section{Abstract}

Objective: This study aimed to study and evaluate the value of platelet-to-lymphocyte ratio (PLR) and neutrophil-to-lymphocyte ratio (NLR) as markers to predict the disease activity and severity in patients with rheumatoid arthritis (RA).

Methods: A total of 139 inpatients with RA were divided into two groups: moderate activity group (3.2<DAS28-CRP score<5.1) and high activity group ( $\geq 5.1)$. The correlation of routine hematological indices with DAS28 and joint ultrasound score (gray-scale ultrasound score, GS-US; power Doppler ultrasound score, PD-US; total ultrasound score, T-US) were analyzed by Pearson's correlation and logistic regression analyses. Receiver operating characteristics (ROC) analysis was performed to compare the efficacy of blood indices, ESR, or CRP in reflecting the disease activity and severity of RA.

Results: The values of PLR, NLR, PD-US, and T-US were significantly different between moderate and high disease activity groups $(p<0.001)$, and PLR was significantly correlated with PD-US and DAS28. Logistic regression analyses showed that PLR was an independent risk factor for disease activity by DAS 28 and joint damage severity by PD-US and T-US. ROC analysis showed that the efficacy of using PLR alone to evaluate the disease activity and joint severity of RA was similar to that of using combined CRP and ESR. The best cut-off value of PLR for predicting high disease activity and high joint severity was determined as 236.6 .

Conclusions: PLR can be used as a marker to predict activity and severity in patients with moderate and high RA disease activity.

\section{Introduction}

Rheumatoid arthritis (RA) is a systemic autoimmune disease characterized by symmetrical, erosive, and progressive synovitis, which is induced by genetic and environmental factors. Inflammation and hyperplasia of the joint synovium could lead to the formation of pannus, which in turn destroy the articular cartilage, subchondral bone, ligaments, and tendons, causing cartilage damage, bone erosion, and destruction of the joint structure. Therefore, it is important to search for reliable indicators to assess disease activity and severity for early prognosis and clinical decision-making.

Currently, the disease activity score in 28 joints (DAS28) is widely used to evaluate the disease activity of RA [1]. Although DAS28 can be calculated by simple software, its performance is still not satisfactory, a few studies reported that clinically significant pain persisted among patients achieving DAS28 remission [2,3]. Traditional X-ray or computed tomography (CT) scans can be easily used to identify bone and joint lesions, thus facilitating the evaluation of disease severity, however, these techniques cannot detect early lesions, and patients are at risk of being exposed to high radiation levels throughout successive tests. On the other hand, gray-scale ultrasound (GSUS) can detect synovitis in real-time (exudation and synovial hypertrophy), and power Doppler ultrasound (PD-US) can display small blood flow signals indicating the degree of severity in the lesion (neovascularization). More importantly, with the help of portable devices and experienced operators, ultrasound can be easily used multiple times to assess disease progression $[4,5]$.

Some routine hematological indices have been proposed as indicators of systemic inflammation in recent years [6-9]. Moreover, routine blood tests do not involve the use of expensive equipment and can be rapidly acquired within a short period, so they are more beneficial in economically backward areas with limited medical equipment and personnel, to reducing the burden of high medical costs for people living in these areas. Several studies have shown that platelet-to-lymphocyte ratio (PLR) combined with neutrophil-to-lymphocyte ratio (NLR) and other hematological indicators can be used to diagnose and evaluate the activity and severity of several rheumatic diseases, such as systemic lupus erythematosus (SLE), Behçet's disease (BD), Takayasu's arteritis (TA), and psoriasis, detect subclinical complications, and monitor the response to anti-inflammatory treatment [10-14]. Studies have also shown NLR and PLR are significantly associated with the disease activity of RA $[15,16]$. To the best of our knowledge, few comparative analyses have been conducted to evaluate the relationship between routine hematological indicators with joint ultrasound score and DAS28.

Our study aimed to assess the correlations between the levels of routine hematological indices with different disease activities and joint ultrasound scores in patients with RA. The purpose was to explore if routine hematological indices can be used to assess disease activity and joint severity of RA in a cost-effective and convenient manner.

\section{Materials And Methods}

\subsection{Patient characteristics and clinical assessments}


This single-center cross-sectional study included 139 inpatients diagnosed with RA according to 2010 European League Against Rheumatism (EULAR) criteria [17]. These patients were admitted into the Department of Rheumatology and Immunology at the Second Xiangya Hospital of Central South University from 01 July,2016 to 30 August,2019. Patients with other rheumatic diseases, severe infections, or malignancies were excluded from this study. Baseline demographic and clinical data were collected retrospectively from their medical records. At the same time, the levels of rheumatoid factors (RF-lgA, RF-IgG, and RF-lgM), levels of anti-cyclic citrullinated peptide antibody (CCP), blood index including mean platelet volume (MPV) and red blood cell distribution width (RDW) were measured, and PLR and NLR were calculated. The joint function was evaluated according to the standards established by the American Academy of Rheumatology [18].

The disease activity of RA was determined using DAS 28 system. To calculate DAS28-ESR and DAS28-CRP, the tender joint count (TJC), swollen joint count (SJC), erythrocyte sedimentation rate (ESR), and C-reactive protein (CRP) levels were measured, and the patients' global assessment (PGA) in visual analog scale (VAS) were applied. Because patients with low disease activity were not admitted to the inpatient ward of the university hospital, the enrolled patients were assumed to have moderate and high disease activity, 49 patients whose DAS28-CRP ranged from 3.2 to 5.1 were categorized into the moderate disease activity group, and 90 patients whose DAS28-CRP was higher than 5.1 were categorized into the high disease activity group. The study was approved by the Institutional Ethics Committee of the Second Xiangya Hospital of Central South University (2020-K011) and was conducted according to the Declaration of Helsinki. Written informed consents were obtained from all participants.

\subsection{Ultrasound evaluation}

To assess joint severity, joint ultrasound scores, including GS-US and PD-US, were measured using a Esote MyLab30Gold color Doppler ultrasound system. The ultrasound test was performed and verified for knee joints and/or wrist joints by the 2 ultrasound physicians who was unknown about patient's clinical data. Joints numbers of ultrasound tests ranged from 1 to 4 were decided by the patients' willingness to bear the medical costs. The total joint ultrasound score (T-US) was calculated by adding GS-US and PD-US for the examined joint and obtaining the average score.

The most frequently used semi-quantitative methods were applied for scoring synovitis [19]. The joint inflammation activity was graded using GS-US as follows: Grade 0, normal joint with no synovial thickening; Grade 1, minimal synovial thickening (filling the angle between the periarticular bones, without bulging over the line linking tops of the bones); Grade 2, synovial thickening bulging over the line linking the tops of the periarticular bones but without extension along the bone diaphysis; Grade 3, synovial thickening bulging over the line linking tops of the periarticular bones and with extension to at least one of the bone diaphysis. Furthermore, arthritis severity was semiquantitatively graded using PD-US as follows: Grade 0 , no flow in the synovium; Grade 1, single vessel signals; Grade 2, confluent vessel signals in less than half of the synovial area; Grade 3, vessel signals in more than half of the synovial area.

\subsection{Statistical analyses}

Statistical analyses were performed using SPSS software version 26.0 (Chicago, IL, USA). To test the normality of the data, the Kolmogorov-Smirnov test was used. The normally distributed variables were expressed as the mean \pm standard deviation $(x \pm s)$. Significant differences between groups were assessed using the t-test. Non-normally distributed variables were represented by the median (upper and lower quartiles), and the Mann-Whitney U-test was used to compare differences between two groups. The data were expressed in frequencies or percentages (\%), and the Chi-square test was used to compare differences between groups. The Pearson's correlation test was used to assess the correlation between routine hematological indices with DAS28 or joint ultrasound scores (PD-US, GS-US, and T-US). Then, Logistic regression analyses were performed to predict the relationship between US or DAS28 and blood indices and to identify the blood indices that best reflect DAS28 and US. Finally, receiver operating characteristics (ROC) analysis was performed to compare the efficacy of blood indices, ESR, or CRP in reflecting the disease activity and severity of RA. Values with two-sided p-values of less than 0.05 were considered statistically significant.

\section{Results}

\subsection{Clinical characteristics of patients in different disease activity groups}

The mean age of the patients was 58 years (23-84 years), and majority patients were females $(100 / 139,71.9 \%)$. There were no significant differences in terms of sex ratio, age, disease duration, number of joints tested, positivity ratio of RF-IgG, IgA, IgM, and antiCCP antibody between patients with moderate and high DAS28 ( $p>0.05)$. The values of clinical indices (SJC, TJC, Joint function, PGA, ESR, and CRP) and ultrasound indices (T-US and PD-US) in patients with high activity RA were higher compared to patients with moderate 
activity RA. Moreover, patients with high disease activity had significantly elevated levels of routine hematological indices, including lymphocyte (L) count, platelet (PLT) count, MPV, PLR, and NLR compared to patients with moderate disease activity $(p<0.05)(T a b l e ~ 1$, Figure1). 
Table 1

Baseline characteristic of 139 patients with rheumatoid arthritis (RA).

\begin{tabular}{|c|c|c|c|c|}
\hline & \multirow{2}{*}{$\begin{array}{l}\text { Total RA patients } \\
(\mathrm{N}=139)\end{array}$} & \multicolumn{3}{|l|}{ Group } \\
\hline & & $\begin{array}{l}\text { RA with Moderate activity } \\
(\mathrm{N}=49)\end{array}$ & $\begin{array}{l}\text { RA with High activity } \\
(\mathrm{N}=90)\end{array}$ & $\mathbf{p}$ \\
\hline Gender(F/M), n (\%) & $100 / 39(71.9 / 28.1)$ & $36 / 13(73.5 / 26.5)$ & 64/26(71.1/28.8) & 0.767 \\
\hline Age of onset, Years & $58.0(52.0,65.0)$ & $54.50(49.5,63.0)$ & $60.0(52.0,66.0)$ & 0.082 \\
\hline $\begin{array}{l}\text { Disease duration, } \\
\text { Months }\end{array}$ & $60.0(12.0,120.0)$ & $60.0(12.0,156.0)$ & $72.0(12.0,120.0)$ & 0.719 \\
\hline \multicolumn{5}{|l|}{ Clinical Parameters } \\
\hline SJC, n & $6.0(2.0,14.0)$ & $2.0(1.0,4.5)$ & $11.0(5.0,21)$. & $0.000^{\star \star \star}$ \\
\hline TJC, n & $10.0(4.0,18.0)$ & $2.0(2.0,5.5)$ & $14.0(8.0,24.0)$ & $0.000^{\star * \star}$ \\
\hline \multirow{2}{*}{$\begin{array}{l}\text { Joint } \\
\text { function(I/II/III/IV), } \\
\mathrm{n}(\%)\end{array}$} & $48 / 37 / 41 / 13$ & $20 / 20 / 9 / 0$ & 28/17/32/13 & \multirow[t]{2}{*}{$0.001^{\star \star}$} \\
\hline & $(34.5 / 26.6 / 29.5 / 9.4)$ & $(40.8 / 40.8 / 18.4 / 0)$ & $(31.1 / 18.8 / 35.5 / 14.1)$ & \\
\hline PGA & $60.0(12.0,120.0)$ & $50.0(32.5,60.0)$ & $70.0(50.0,80.0)$ & $0.000^{\star \star \star}$ \\
\hline $\mathrm{ESR}, \mathrm{mm} / \mathrm{h}$ & $80.0(52.0,94.5)$ & $60.0(40.0,86.5)$ & $86.0(59.0,100.0)$ & $0.001^{\star \star}$ \\
\hline CRP, mg/dl & $49.6(20.7,90.1)$ & $29.3(11.4,76.4)$ & $58.6(27.6,101.0)$ & $0.010^{*}$ \\
\hline $\begin{array}{l}\text { RF-IgG }{ }^{-} / R^{2}-I^{\prime g G}{ }^{+}, n \\
(\%)\end{array}$ & 51/88(36.7/63.3) & 18/31(36.7/63.3) & 33/57(36.7/63.3) & 0.994 \\
\hline $\begin{array}{l}\text { RF-lgA }{ }^{-} / R^{2}-\lg A^{+}, n \\
(\%)\end{array}$ & 65/74(46.8/53.2) & 21/28(42.8/57.1) & $44 / 46(48.8 / 51.1)$ & 0.496 \\
\hline $\begin{array}{l}\text { RF-IgM }{ }^{-} / R F-I g M^{+} \\
n(\%)\end{array}$ & 41/98(29.5/70.5) & 13/36(26.5/73.5) & 28/62(31.1/68.9) & 0.572 \\
\hline $\begin{array}{l}\text { anti-CCP }{ }^{-} / \text {anti- } \\
\mathrm{CCP}^{+}, \mathrm{n}(\%)\end{array}$ & $22 / 117(15.8 / 84.1)$ & 8/41(16.3/83.7) & 14/76(15.6/84.4) & 0.905 \\
\hline $\mathrm{Hb}, \mathrm{g} / \mathrm{L}$ & $102.6 \pm 18.9$ & $106.9 \pm 19.8$ & $100.2 \pm 18.0$ & $0.045^{*}$ \\
\hline WBC, $\times 10^{9} / \mathrm{L}$ & $6.8(5.2,8.5)$ & $6.3(5.4,7.8)$ & $6.9(5.0,8.7)$ & 0.726 \\
\hline $\mathrm{N}, \times 10^{9} / \mathrm{L}$ & $4.8(3.5,6.6)$ & $4.2(3.4,5.6)$ & $4.9(3.5,6.9)$ & 0.260 \\
\hline $\mathrm{L}, \times 10^{9} / \mathrm{L}$ & $1.4(1.1,1.6)$ & $1.6(1.2,1.9)$ & $1.3(0.9,1.6)$ & $0.003^{\star \star}$ \\
\hline Plt, $\times 10^{9} / \mathrm{L}$ & $320.0(265.5,411.0)$ & 293.5(249.0,382.0) & $350.5(276.0,442.0)$ & $0.022^{*}$ \\
\hline RDW, \% & $14.6 \pm 2.1$ & $14.5 \pm 2.3$ & $14.7 \pm 2.0$ & 0.468 \\
\hline MPV, fL & $10.3 \pm 1.2$ & $10.6 \pm 1.3$ & $10.1 \pm 1.1$ & 0.090 \\
\hline NLR & $3.4(2.4,4.9)$ & $2.8(2.2,3.6)$ & $3.7(2.6,5.8)$ & $0.003^{\star \star}$ \\
\hline PLR & 244.8(190.0, 322.7) & 207.6(167.7, 244.8) & $282.2(215.4,378.5)$ & $0.000^{\star \star \star}$ \\
\hline \multicolumn{5}{|l|}{ Ultrasound Parameters } \\
\hline \multicolumn{5}{|c|}{$\star p<0.05, * * p<0.005, * \star * p<0.001$} \\
\hline \multicolumn{5}{|c|}{$\begin{array}{l}\text { Abbreviations: CCP, anti-cyclic citrullinated peptide antibody; CRP, C-reactive protein; DAS28, disease activity score in } 28 \text { joints; ESR, } \\
\text { erythrocyte sedimentation rate; GS-US, grey-scale ultrasound score; Hb, hemoglobin; L, lymphocyte; MPV, mean platelet volume; N, } \\
\text { neutrophil; NLR, neutrophil-to-lymphocyte ratio; PD-US, power Doppler ultrasound score; PGA, patient global assessment; PLR, platelet- } \\
\text { to-lymphocyte ratio; PIt, platelet; RDW, red blood cell distribution width; RF, rheumatoid factor; SJC, swollen joint count; TJC, tender } \\
\text { joint count; T-US, total ultrasound Score; WBC, white blood cell. }\end{array}$} \\
\hline
\end{tabular}




\begin{tabular}{|c|c|c|c|c|}
\hline & \multirow{2}{*}{$\begin{array}{l}\text { Total RA patients } \\
(\mathrm{N}=139)\end{array}$} & \multicolumn{3}{|l|}{ Group } \\
\hline & & $\begin{array}{l}\text { RA with Moderate activity } \\
(\mathrm{N}=49)\end{array}$ & $\begin{array}{l}\text { RA with High activity } \\
(\mathrm{N}=90)\end{array}$ & $\mathrm{p}$ \\
\hline \multirow{2}{*}{$\begin{array}{l}\text { Patients' number } \\
\text { testing } 1 / 2 / 3 / 4 \\
\text { joints, } \mathrm{n}(\%)\end{array}$} & $64 / 60 / 12 / 3$ & $23 / 21 / 4 / 1$ & $41 / 39 / 8 / 2$ & \multirow[t]{2}{*}{0.993} \\
\hline & $(46.0 / 43.1 / 8.6 / 2.1)$ & $(46.9 / 42.8 / 8.2 / 2.0)$ & $(45.5 / 43.3 / 8.9 / 2.2)$ & \\
\hline $\begin{array}{l}\text { Total joints } \\
\text { numbers tested, } n\end{array}$ & 232 & 81 & 151 & - \\
\hline \multirow{2}{*}{$\begin{array}{l}\text { PD-US joints } \\
\text { number scoring } \\
0 / 1 / 2 / 3, \mathrm{n}(\%)\end{array}$} & $35 / 37 / 159 / 1$ & $20 / 14 / 47 / 0$ & $15 / 23 / 112 / 1$ & \multirow[t]{2}{*}{$0.016^{*}$} \\
\hline & $(15.1 / 15.9 / 68.5 / 0.4)$ & $(24.7 / 17.3 / 58.0 / 0)$ & $(9.9 / 15.2 / 74.2 / 0.6)$ & \\
\hline PD-US scores & $2.0(1.0,2.0)$ & $1.5(1.0,2.0)$ & $2.0(1.5,2.0)$ & $0.010^{*}$ \\
\hline \multirow{2}{*}{$\begin{array}{l}\text { GS-US joints } \\
\text { number scoring } \\
0 / 1 / 2 / 3, \mathrm{n}(\%)\end{array}$} & $0 / 0 / 142 / 90$ & $0 / 0 / 54 / 27$ & $0 / 0 / 88 / 63$ & \multirow[t]{2}{*}{0.211} \\
\hline & $(0 / 0 / 61.2 / 38.8)$ & $(0 / 0 / 66.7 / 33.3)$ & $(0 / 0 / 58.3 / 41.7)$ & \\
\hline GS-US scores & $2.0(2.0,3.0)$ & $2.0(2.0,3.0)$ & $2.33(2.0,3.0)$ & 0.288 \\
\hline \multirow{2}{*}{$\begin{array}{l}\text { T-US joints } \\
\text { number scoring } \\
0 / 1 / 2 / 3 / 4 / 5 / 6, \mathrm{n} \\
(\%)\end{array}$} & 0/0/35/33/78/85/1 & $0 / 0 / 20 / 14 / 20 / 27 / 0$ & 0/0/15/19/58/58/1 & \multirow[t]{2}{*}{$0.015^{*}$} \\
\hline & $(0 / 0 / 15.1 / 14.2 / 33.6 / 36.6 / 0.4)$ & $(0 / 0 / 24.7 / 17.3 / 24.7 / 33.3 / 0)$ & $(0 / 0 / 9.9 / 12.6 / 38.4 / 38.4 / 0.6)$ & \\
\hline T-US scores & $4.0(3.5,5.0)$ & $4.0(3.0,5.0)$ & $4.0(3.5,5.0)$ & $0.039^{*}$ \\
\hline \multicolumn{5}{|l|}{ Disease activity } \\
\hline DAS28-ESR & $6.2 \pm 1.3$ & $4.8 \pm 0.7$ & $7.0 \pm 0.9$ & $0.000^{\star \star \star}$ \\
\hline DAS28-CRP & $5.6 \pm 1.3$ & $4.2 \pm 0.6$ & $6.4 \pm 0.9$ & $0.000^{\star \star \star}$ \\
\hline \multicolumn{5}{|c|}{$\star p<0.05, * \star p<0.005, * \star * p<0.001$} \\
\hline \multicolumn{5}{|c|}{$\begin{array}{l}\text { Abbreviations: CCP, anti-cyclic citrullinated peptide antibody; CRP, C-reactive protein; DAS28, disease activity score in } 28 \text { joints; ESR, } \\
\text { erythrocyte sedimentation rate; GS-US, grey-scale ultrasound score; Hb, hemoglobin; L, lymphocyte; MPV, mean platelet volume; N, } \\
\text { neutrophil; NLR, neutrophil-to-lymphocyte ratio; PD-US, power Doppler ultrasound score; PGA, patient global assessment; PLR, platelet- } \\
\text { to-lymphocyte ratio; PIt, platelet; RDW, red blood cell distribution width; RF, rheumatoid factor; SJC, swollen joint count; TJC, tender } \\
\text { joint count; T-US, total ultrasound score; WBC, white blood cell. }\end{array}$} \\
\hline
\end{tabular}

\subsection{Correlation analysis}

\subsubsection{Correlation of routine hematological indices and DAS28}

The correlation between routine hematological indices (Hb, WBC, N, L, PLT, RDW, MPV, NLR, and PLR) and DAS28-ESR or DAS28-CRP was assessed by Pearson's correlation analysis. PLT, NLR, and PLR were found significantly positively correlated with both DAS28-ESR and DAS28-CRP, while Hb, L, and MPV were significantly negatively correlated with DAS28 (Figure 2-A, Figure 2-B, Table 2). However, no significantly correlations were observed between WBC, N, RDW, and DAS28. 
Table 2

Association between routine hematological indices with disease activity score in 28 joints (DAS28) and ultrasound scores.

\begin{tabular}{|c|c|c|c|c|c|c|c|c|c|c|c|}
\hline & & & $\mathrm{Hb}$ & WBC & $\mathbf{N}$ & $\mathrm{L}$ & Plt & RDW & MPV & NLR & PLR \\
\hline \multirow{5}{*}{$\begin{array}{l}\text { DAS28- } \\
\text { ESR }\end{array}$} & \multirow[t]{2}{*}{ Pearson } & $r$ & $-0.186^{*}$ & 0.009 & 0.077 & $-0.236^{\star \star}$ & $0.261^{* *}$ & 0.021 & $-0.182^{*}$ & $0.216^{\star}$ & $0.405^{\star \star \star}$ \\
\hline & & $p$ & 0.029 & 0.918 & 0.366 & 0.005 & 0.002 & 0.812 & 0.037 & 0.011 & 0.000 \\
\hline & \multirow[t]{3}{*}{ Logistic } & OR & & / & / & $\mathrm{N} / \mathrm{A}$ & 0.997 & / & 0.830 & 1.147 & $1.009 *$ \\
\hline & & $(95 \% \mathrm{Cl})$ & $\begin{array}{l}(0.961- \\
1.011)\end{array}$ & & & & $\begin{array}{l}(0.992- \\
1.003)\end{array}$ & & $\begin{array}{l}(0.552- \\
1.247)\end{array}$ & $\begin{array}{l}(0.822- \\
1.601)\end{array}$ & $\begin{array}{l}(1.001- \\
1.018)\end{array}$ \\
\hline & & $p$ & 0.269 & / & / & $\mathrm{N} / \mathrm{A}$ & 0.326 & / & 0.370 & 0.419 & 0.033 \\
\hline \multirow{5}{*}{$\begin{array}{l}\text { DAS28- } \\
\text { CRP }\end{array}$} & \multirow[t]{2}{*}{ Pearson } & $r$ & $-0.174^{\star}$ & 0.066 & 0.135 & $-0.219^{\star}$ & $0.263^{\star *}$ & 0.053 & -0.149 & $0.256^{\star \star}$ & $0.393^{\star \star \star}$ \\
\hline & & $p$ & 0.041 & 0.442 & 0.114 & 0.010 & 0.002 & 0.541 & 0.089 & 0.002 & 0.000 \\
\hline & \multirow[t]{3}{*}{ Logistic } & OR & 0.988 & / & / & $\mathrm{N} / \mathrm{A}$ & 1.000 & / & / & 1.160 & $1.007 *$ \\
\hline & & $(95 \% \mathrm{Cl})$ & $\begin{array}{l}(0.998- \\
1.010)\end{array}$ & & & & $\begin{array}{l}(0.996- \\
1.004)\end{array}$ & & & $\begin{array}{l}(0.892-1 . \\
509)\end{array}$ & $\begin{array}{l}(1.001- \\
1.013)\end{array}$ \\
\hline & & $p$ & 0.292 & / & / & $\mathrm{N} / \mathrm{A}$ & 0.971 & / & / & 0.269 & 0.025 \\
\hline \multirow[t]{5}{*}{ PD-US } & \multirow[t]{2}{*}{ Pearson } & $r$ & -0.074 & 0.097 & 0.134 & -0.155 & $0.238^{\star *}$ & -0.041 & -0.112 & $0.212^{*}$ & $0.310^{\star \star \star}$ \\
\hline & & $p$ & 0.389 & 0.256 & 0.116 & 0.068 & 0.005 & 0.636 & 0.202 & 0.012 & 0.000 \\
\hline & \multirow[t]{3}{*}{ Logistic } & OR & / & / & / & / & 1.001 & / & / & 0.969 & $1.016^{*}$ \\
\hline & & $(95 \% \mathrm{Cl})$ & & & & & $\begin{array}{l}(0.994- \\
1.008)\end{array}$ & & & $\begin{array}{l}(0.647- \\
1.452)\end{array}$ & $\begin{array}{l}(1.003- \\
1.028)\end{array}$ \\
\hline & & $p$ & / & / & / & / & 0.841 & / & / & 0.879 & 0.012 \\
\hline \multirow[t]{5}{*}{ GS-US } & \multirow[t]{2}{*}{ Pearson } & $r$ & 0.027 & 0.107 & 0.112 & 0.023 & 0.108 & 0.008 & -0.091 & 0.078 & 0.040 \\
\hline & & $p$ & 0.756 & 0.211 & 0.190 & 0.789 & 0.205 & 0.924 & 0.298 & 0.360 & 0.636 \\
\hline & \multirow[t]{3}{*}{ Logistic } & OR & / & / & / & / & / & / & / & / & / \\
\hline & & $(95 \% \mathrm{Cl})$ & & & & & & & & & \\
\hline & & $p$ & / & / & / & / & / & / & / & / & / \\
\hline \multirow[t]{5}{*}{ T-US } & \multirow[t]{2}{*}{ Pearson } & $r$ & -0.023 & 0.122 & 0.144 & -0.059 & $0.212^{\star}$ & -0.012 & -0.135 & 0.157 & $0.197^{\star}$ \\
\hline & & $\mathrm{p}$ & 0.786 & 0.152 & 0.091 & 0.489 & 0.012 & 0.893 & 0.123 & 0.065 & 0.020 \\
\hline & \multirow[t]{3}{*}{ Logistic } & & / & / & / & / & 1.001 & / & / & I & $1.016^{\star \star}$ \\
\hline & & $(95 \% \mathrm{Cl})$ & & & & & $\begin{array}{l}(0.993- \\
1.008)\end{array}$ & & & & $\begin{array}{l}(1.005- \\
1.028)\end{array}$ \\
\hline & & $p$ & / & / & / & / & 0.876 & / & / & / & 0.006 \\
\hline \multicolumn{12}{|c|}{ 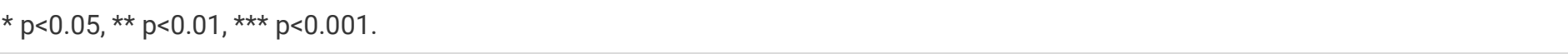 } \\
\hline \multicolumn{12}{|c|}{$\begin{array}{l}\text { / : Because there were no significant differences in Pearson's correlation analysis, Logistic analysis was not applied. N/A: Because } \\
\text { strong multicollinearity with other independent variables were observed, L was not applied with Logistic analysis. }\end{array}$} \\
\hline \multicolumn{12}{|c|}{$\begin{array}{l}\text { Abbreviations: CRP, C-reactive protein; DAS28, disease activity score in } 28 \text { joints; ESR, erythrocyte sedimentation rate; GS-US, grey- } \\
\text { scale ultrasound score; Hb, hemoglobin; L, lymphocyte; MPV, mean platelet volume; N, neutrophil; NLR, neutrophil-to-lymphocyte ratio; } \\
\text { PD-US, power Doppler ultrasound score; PLR, platelet-to-lymphocyte ratio; Plt, platelet; RDW, red blood cell distribution width; T-US, } \\
\text { total ultrasound score; WBC, white blood cell. }\end{array}$} \\
\hline
\end{tabular}

Binary logistic regression (LR) analysis was performed to assess which of above markers were risk factors associated with the DAS28 grade. Because there was collinearity between lymphocyte with other indicators (tolerance $<0.2$ ), lymphocyte was excluded outside of LR analysis, therefore the variables included in the LR analysis were Hb, PLT, MPV, NLR, and PLR. The results confirmed that PLR, which 
correlates with DAS28-ESR (odds ratio $[O R]=1.009, p=0.033$ ) and DAS28-CRP (OR=1.007, $p=0.025)$, was an independent risk factor for the disease activity of RA (Table 2).

\subsubsection{Correlation of routine hematological indices with ultrasound scores}

The correlation between routine hematological indices (Hb, WBC, N, L, PLT, RDW, MPV, NLR, and PLR) and joint ultrasound was assessed by Pearson's correlation analysis. Results of Pearson's correlation analysis showed that PLT, NLR, and PLR were significantly positively correlated with PD-US meanwhile PLT and PLR were significantly positively correlated with T-US $(p<0.05)$. However there was no hematological index was found to be significantly correlated with GS-US (Figure 2-C, Figure 2-D, Figure 2-E, Table 2).

Binary logistic regression (LR) analysis was performed to assess which of above markers was risk factors associated with the joint ultrasound grade. The variables included in the LR analysis of PD-US grade $(\geq 1,<1)$ were PLT, NLR, and PLR, while PLT and PLR were included in the LR analysis of T-US grade $(\geq 3,<3)$. All variables were numerical and were entered simultaneously. Results of collinearity diagnostics indicated no collinearity between the variables (tolerance $>0.2$ and VIF $<5$ ). The results confirmed that PLR, which correlates with ultrasound scores including PD-US (OR=1.016, $p=0.012)$ and T-US, $(O R=1.016, p=0.006)$, was an independent risk factor for the disease severity of RA (Table 2 ).

\subsection{Determination of the best cut-off value of PLR}

ROC analysis was performed to compare the efficacy of ESR, CRP, PLR, ESR+CRP, PLR+ESR, PLR+CRP, and PLR+ESR+CRP in assessing the activity of RA (DAS28). Moreover, the efficacy of these indices was compared in evaluating the severity of RA (ultrasound scores).

Results of ROC curve analysis of PLR with DAS28-ESR as a standard revealed that PLR could reflect disease activity (DAS28-ESR) efficiently ( $A U C=0.734, p<0.000)$. The cut-off value of PLR was 242.7 , with high sensitivity $(0.61)$ and specificity $(0.84)$. Comparison of the ROC values of PLR+ESR, PLR+CRP, and PLR+ESR+CRP with the ROC value of PLR alone revealed that the area under the ROC curve (AUROC) was not significantly improved $(0.755,0.734,0.764$, and $0.734, p>0.05)$ (Figure $3 \mathrm{~A}$ ). Then, by analyzing the ROC curve of PLR with that of DAS28-CRP as a standard, we found that PLR could reflect disease activity (DAS28-CRP) efficiently $(A U C=0.718, p<0.000)$. The cut-off value of PLR was 247.3, with high sensitivity (0.61) and specificity (0.78). Comparison of the ROC values of PLR+ESR, PLR+CRP, and PLR+ESR+CRP with the ROC value of PLR alone revealed that the AUROC was not significantly improved $(0.726,0.715,0.728$ and $0.718, p>0.05)$ (Figure $3 B$ ). Therefore, PLR alone is a reliable index with good diagnostic efficacy for predicting disease activity.

Similarly, by comparing the ROC curve of PLR with that of PD-US as a standard, we found that PLR could reflect RA severity (PD-US) efficiently (AUC=0.789, $p<0.000$ ). The cut-off value of PLR was 228.1 , with high sensitivity $(0.61)$ and specificity $(0.87)$. Comparison of the ROC values of PLR+ESR, PLR+CRP, and PLR+ESR+CRP with the ROC value of PLR alone revealed that the AUROC was not significantly improved $(0.808,0.819,0.823$, and $0.789, p>0.05)$ (Figure $3 C)$. Then, by comparing the ROC curve of PLR with that of T-US as a standard, we found that PLR could reflect RA severity (T-US) efficiently (AUC=0.799, $p<0.000$ ). The cut-off value of PLR was 228.1, with high sensitivity (0.61) and specificity (0.86). Comparison of the ROC values of PLR+ESR, PLR+CRP, and PLR+ESR+CRP with the ROC value of PLR alone revealed that the AUROC was not significantly improved $(0.807,0.827,0.824$ and $0.799, p>0.05)$ (Figure 3D). Therefore, PLR alone is a reliable index with good diagnostic efficacy for predicting disease severity.

According to the optimal cut-off value of PLR (average $=236.6$ ), the patients were divided into low PLR and high PLR groups. Observing the changes in the clinical parameters of the two different PLR groups, DAS28, T-US, and PD-US were significantly different between the two groups $(\mathrm{p}<0.001)($ Table 3$)$. 
Table 3

Comparison of the characteristics of disease activity score in 28 joints (DAS28) and ultrasound score between the two groups based on the cut-off value of platelet-to-lymphocyte ratio (PLR).

\begin{tabular}{|llll|}
\hline & PLR<236.6 $(n=66)$ & PLR>236.6 $(n=73)$ & P \\
\hline DAS28-ESR & $5.7 \pm 1.4$ & $6.6 \pm 1.1$ & $0.000^{\star *}$ \\
\hline DAS28-CRP & $5.2 \pm 1.4$ & $6.0 \pm 1.1$ & $0.000^{\star *}$ \\
\hline T-US & $4.0(3.0,5.0)$ & $4.0(4.0,5.0)$ & $0.001^{\star *}$ \\
\hline GS-US & $2.0(2.0,3.0)$ & $2.3(2.0,3.0)$ & 0.507 \\
\hline PD-US & $1.5(1.0,2.0)$ & $2.0(1.6,2.0)$ & $0.024^{\star}$ \\
\hline * p<0.05, **p<0.001. & & \\
\hline $\begin{array}{l}\text { Abbreviations: CRP, C-reactive protein; DAS28, disease activity score in 28 joints; ESR, erythrocyte sedimentation rate; GS-US, grey- } \\
\text { scale ultrasound score; PLR, platelet-to-lymphocyte ratio; T-US, total ultrasound score. }\end{array}$ \\
\hline
\end{tabular}

\section{Discussion}

Currently, the DAS system and imaging analysis, including ultrasonography and radiology, are widely used to evaluate the activity and severity of RA. However, there is a lack of a reliable hematological index that can be used to assess RA activity and severity with a highly convenient and cost-effective manner during routine hematological tests which would benefit developing countries with poor health infrastructure and lack of access to sophisticated imaging techniques. Therefore, we aimed to explore valuable indices used in simple routine blood tests that may evaluate both the activity and severity of RA. In this single-center retrospective study of 139 patients, we found that PLR was significantly correlated with the disease activity and joint severity of patients with RA, similar to DAS28 and ultrasound scores. Analysis of ROC indicated that PLR had a higher sensitivity and specificity than ESR and CRP in predicting disease activity and severity. As an economical and convenient clinical parameter, PLR can be a simple and reliable index for predicting activity and severity in moderate and high disease activity of RA.

In recent years, some routine hematological indices have been proposed as systemic inflammatory indices in rheumatic diseases. Several studies have shown that patients with systemic lupus erythematosus (SLE) have significantly increased NLR and PLR, which were positively correlated with the SLE disease activity index (SLEDAI) and that NLR may be an indicator for monitoring disease activity and reflecting renal involvement in patients with SLE [20]. Some studies have shown that the Psoriasis Area and Severity Index scores of psoriasis patients were significantly higher in the NLR-high and PLR-high subgroups than that in the NLR-low and PLR-low subgroups, The NLR-high subgroup showed higher CRP levels [21]. There were several studies have shown that NLR and PLR were significantly correlated with DAS28 in patients with RA and that PLR may be used as a new biomarker for predicting and diagnosing RA and RA-ILD $[22,23]$. There were a lot of papers showing that platelet activation and high platelet reactivity induce chronic inflammation through the production of thrombopoietin and cytokines. Platelets have been reported to participate in immune regulation and cytokine production depending on their activation state [24-30]. PLT and PLR was positively correlated with DAS28 and PLR was found as an inflammatory factor that can predict disease activity in our study. Moreover, our study showed that the count of lymphocytes in peripheral blood was negatively correlated with disease activity. The decrease in the count of peripheral lymphocytes may be caused by the continuous accumulation of lymphocytes in inflammatory joints and the high levels of early apoptosis markers in lymphocytes, which may be related to autoimmunity [31, 32]. Our findings were consistent with the above mechanism of RA.

Histologically, RA is characterized by inflammatory cell infiltration and the formation of rich vascular pannus. Angiogenesis starts at a very early stage of RA under the influence of various inflammatory cytokines, chemokines, and growth factors [33]. High levels of Ang-1, Ang-2, VEGF, and MMP-3 in the serum and synovium of patients with RA reflect disease activity [34,35]. Compared to composite disease activity indices, PD-US vascularity grading is better correlated with some markers of angiogenesis and inflammation [3]. In recent years, PD-US has been considered an important tool to detect early synovial inflammation and monitor treatment response [36, 37]. One clinical report in 2020 had shown that NLR and PLR were significantly positively correlated with all US parameters of disease activity (gray-scale US, power Doppler US, and global scores) [39]. In our study, the results demonstrated that PLR was an independent risk factor of ultrasound scores (PD-US and T-US). Mechanism of pathological process could help us to understand the finding from ultrasound. In inflamed joints, activated circulating platelets can interact with erythrocytes, neutrophils, and lymphocytes in the vessel lumen at sites of vascular damage [40,41]. Platelets also promote the re-aggregation of neutrophils into the RA synovial fluid. Furthermore, platelets can interact with neutrophils via the expression of platelet-type lipoxygenase and the activation of the eicosanoid pathway, thus aggravating

Page 9/15 
synovitis [28]. Additionally, platelets and their activated products can activate the coagulation and fibrinolysis system of the local synovium of RA, induce VEGF secretion in inflammatory sites, increase vascular permeability, promote neovascularization, increase the expression of MMP-3, destroy and decompose cartilage matrix, and aggravate the development of RA [26, 27]. All these studies provided strong evidence on the correlation between PLR and severity of RA (PD-US).

In clinical application, different scores based on different numbers of joints subjected to ultrasonic detection have been proposed, such as 28-joint, 12-joint, and 7-joint ultrasound scores [42-44], to effectively evaluate the severity of RA. However, examination of a large number of joints is associated with a longer detection time and higher medical costs, which greatly reduce the convenience of ultrasonography as a disease activity assessment tool in the real world. Several studies have shown that detecting a smaller number of joints is equally effective in assessing the disease activity and severity of RA [45]. In this study, we selected either the wrist or knee joint of patients with RA and identified the tested site guided by the patients' willingness, which was in line with the core principle of a patientreported outcome (PRO). Based on this basic principle, only the most affected joints can be selected and subjected to ultrasonography instead of examining pre-arranged numbers of joints. Therefore, patients can avoid undergoing an ultrasonic examination of a large number of joints, thus effectively reducing the time and medical costs associated with ultrasonography.

There were some limitations to this study. First, this was a cross-sectional study with a small sample size. Second, all enrolled patients come from hospitalized ones with moderate or high disease activity and there were no low disease activity patients which limited. We could not directly conclude that PLR was marker for the activity and severity of RA. Finally, longitudinal studies should be conducted to evaluate the clinical value of PLR in the prediction of treatment effects and relapse of RA in the future.

\section{Conclusion}

In this study, we mainly studied the correlations between routine hematological indices with the disease activity score and joint ultrasound score in patients with RA. We attempted to study if routine hematological indices could accurately assess the activity and joint severity of RA in a cost-effective and convenient manner. The results showed that PLR, NLR, PD-US, and T-US were significantly different between moderate and high disease activity groups. PLR, a clinical index routinely measured in hematological tests, was significantly correlated with PD-US and DAS28. Moreover, the results of logistic regression analysis showed that PLR is an independent risk factor for disease activity and joint severity in RA. The efficacy of using PLR alone to evaluate disease activity and severity was similar to that of using combined CRP and ESR. PLR is a blood parameter that can be economically and conveniently assessed during routine blood tests in developing countries, PLR can be a simple and reliable index for predicting the activity and severity in moderate and high disease activity of RA.

\section{Abbreviations}

CRP, C-reactive protein; DAS28, disease activity score in 28 joints; ESR, erythrocyte sedimentation rate; GS-US, grey-scale ultrasound score; $\mathrm{Hb}$, hemoglobin; L, lymphocyte; MPV, mean platelet volume; N, neutrophil; NLR, neutrophil-to-lymphocyte ratio; PD-US, power Doppler ultrasound score; PLR, platelet-to-lymphocyte ratio; PIt, platelet; RDW, red blood cell distribution width; T-US, total ultrasound score; WBC, white blood cell.

\section{Declarations}

1. Acknowledgements

Not applicable.

\section{Authors' contributions}

FL conceived and designed the study. BLC, QZ, SL and YG collected clinical data. PJW and JT performed the ultrasound tests. BLC and FL performed the statistical analysis. BLC wrote the draft of the manuscript. FL and XX critically reviewed the manuscript. All authors have read and approved the final version of the manuscript.

3. Funds: This study was supported by the National Natural Science Foundation of China $(81571599,81873882)$; National foundation of HUNAN province (2018JJ2588,2021 JJ30934); Hunan Provincial Health Committee 225 Talent Project (2019-196); Educational Fund of Hunan Provincial Finance Department (2021-22-2050205). 
4. Data availability statement

Additional data are available upon reasonable request. The datasets used and/or analyzed in the present study are available from the corresponding author on reasonable request.

5. Ethics approval and consent to participate

The study was approved by the Institutional Ethics Committee of the Second Xiangya Hospital of Central South University (2020-K011) and was conducted according to the Declaration of Helsinki. Written informed consents were obtained from all participants.

6. Consent for publication

Not applicable.

7. Competing interests

The authors have no conflicts of interest to declare.

\section{References}

1. Anderson J, Caplan L, Yazdany J, et al. Rheumatoid Arthritis Disease Activity Measures: American College of Rheumatology Recommendations for Use in Clinical Practice. Arthritis Care \& Research. 2012;64(5):640-647.

2. Lee YC, Cui J, Lu B, et al. Pain persists in DAS28 rheumatoid arthritis remission but not in ACR/EULAR remission: a longitudinal observational study. Arthritis research \& therapy. 2011;13(3):R83.

3. Misra S, Mondal S, Chatterjee S, Guin A, Sinhamahapatra P, Ghosh A. Association of Angiogenic and Inflammatory Markers with Power Doppler Ultrasound Vascularity Grade and DAS28-CRP in Early Rheumatoid Arthritis: A Comparative Analysis. BioMed research international. 2018;2018:6906374.

4. Walther M, Harms H, Krenn V, Radke S, Faehndrich TP, Gohlke F. Correlation of power Doppler sonography with vascularity of the synovial tissue of the knee joint in patients with osteoarthritis and rheumatoid arthritis. Arthritis and rheumatism. 2001;44(2):331338.

5. Reiche BE, Ohrndorf S, Feist E, Messerschmidt J, Burmester GR, Backhaus M. Usefulness of power Doppler ultrasound for prediction of re-therapy with rituximab in rheumatoid arthritis: a prospective study of longstanding rheumatoid arthritis patients. Arthritis Care Res (Hoboken). 2014;66(2):204-216.

6. Zhang Y, Lu JJ, Du YP, Feng CX, Wang LQ, Chen MB. Prognostic value of neutrophil-to-lymphocyte ratio and platelet-to-lymphocyte ratio in gastric cancer. Medicine. 2018;97(12):e0144.

7. Li X, Shen J, Lu Z, Chen M, Fang X, Wang G. High neutrophil-to-lymphocyte ratio is associated with increased carotid artery intimamedia thickness in type 2 diabetes. Journal of diabetes investigation. 2017;8(1):101-107.

8. Cho JH, Cho HJ, Lee HY, et al. Neutrophil-Lymphocyte Ratio in Patients with Acute Heart Failure Predicts In-Hospital and Long-Term Mortality. Journal of clinical medicine. 2020;9(2).

9. Mae Y, Takata T, Ida A, et al. Prognostic Value of Neutrophil-To-Lymphocyte Ratio and Platelet-To-Lymphocyte Ratio for Renal Outcomes in Patients with Rapidly Progressive Glomerulonephritis. Journal of clinical medicine. 2020;9(4).

10. Ma L, Zeng A, Chen B, Chen Y, Zhou R. Neutrophil to lymphocyte ratio and platelet to lymphocyte ratio in patients with systemic lupus erythematosus and their correlation with activity: A meta-analysis. International immunopharmacology. 2019;76:105949.

11. Cho JH, Cho HJ, Lee HY, et al. Neutrophil-Lymphocyte Ratio in Patients with Acute Heart Failure Predicts In-Hospital and Long-Term Mortality. Journal of clinical medicine. 2020;9(2).

12. Pan L, Du J, Li T, Liao H. Platelet-to-lymphocyte ratio and neutrophil-to-lymphocyte ratio associated with disease activity in patients with Takayasu's arteritis: a case-control study. BMJ open. 2017;7(4):e014451.

13. Paliogiannis P, Satta R, Deligia G, et al. Associations between the neutrophil-to-lymphocyte and the platelet-to-lymphocyte ratios and the presence and severity of psoriasis: a systematic review and meta-analysis. Clinical and experimental medicine. 2019;19(1):3745.

14. Gasparyan AY, Ayvazyan L, Mukanova U, Yessirkepov M, Kitas GD. The Platelet-to-Lymphocyte Ratio as an Inflammatory Marker in Rheumatic Diseases. Annals of laboratory medicine. 2019;39(4):345-357. 
15. Uslu AU, Küçük A, Şahin A, et al. Two new inflammatory markers associated with Disease Activity Score-28 in patients with rheumatoid arthritis: neutrophil-lymphocyte ratio and platelet-lymphocyte ratio. International journal of rheumatic diseases. 2015;18(7):731-735.

16. Fu H, Qin B, Hu Z, et al. Neutrophil- and platelet-to-lymphocyte ratios are correlated with disease activity in rheumatoid arthritis. Clinical laboratory. 2015;61(3-4):269-273.

17. Aletaha D, Neogi T, Silman AJ, et al. 2010 rheumatoid arthritis classification criteria: an American College of Rheumatology/European League Against Rheumatism collaborative initiative. Annals of the rheumatic diseases. 2010;69(9):15801588.

18. Prevoo ML, van 't Hof MA, Kuper HH, van Leeuwen MA, van de Putte LB, van Riel PL. Modified disease activity scores that include twenty-eight-joint counts. Development and validation in a prospective longitudinal study of patients with rheumatoid arthritis. Arthritis and rheumatism. 1995;38(1):44-48.

19. D'Agostino MA, Terslev L, Aegerter P, et al. Scoring ultrasound synovitis in rheumatoid arthritis: a EULAR-OMERACT ultrasound taskforce-Part 1: definition and development of a standardised, consensus-based scoring system. RMD open. 2017;3(1):e000428.

20. Wang L, Wang C, Jia X, Yang M, Yu J. Relationship between Neutrophil-to-Lymphocyte Ratio and Systemic Lupus Erythematosus: A Meta-analysis. Clinics (Sao Paulo, Brazil). 2020;75:e1450.

21. Asahina A, Kubo N, Umezawa Y, Honda H, Yanaba K, Nakagawa H. Neutrophil-lymphocyte ratio, platelet-lymphocyte ratio and mean platelet volume in Japanese patients with psoriasis and psoriatic arthritis: Response to therapy with biologics. The Journal of dermatology. 2017;44(10):1112-1121.

22. Chen Q, Chen DY, Xu XZ, Liu YY, Yin TT, Li D. Platelet/Lymphocyte, Lymphocyte/Monocyte, and Neutrophil/Lymphocyte Ratios as Biomarkers in Patients with Rheumatoid Arthritis and Rheumatoid Arthritis-Associated Interstitial Lung Disease. Medical science monitor: international medical journal of experimental and clinical research. 2019;25:6474-6481.

23. Erre GL, Paliogiannis P, Castagna F, et al. Meta-analysis of neutrophil-to-lymphocyte and platelet-to-lymphocyte ratio in rheumatoid arthritis. European journal of clinical investigation. 2019;49(1):e13037.

24. Wang F, Wang NS, Yan CG, Li JH, Tang LQ. The significance of platelet activation in rheumatoid arthritis. Clinical rheumatology. 2007;26(5):768-771.

25. Rui-Kai Z, Jian L. Effects of xinfeng capsules on expression of platelet granule membrane protein 140 and platelet cluster of differentiation 40 ligand in peripheral blood of adjuvant arthritis rats. International journal of rheumatology. 2012;2012:139696.

26. Duchez AC, Boudreau LH, Naika GS, et al. Platelet microparticles are internalized in neutrophils via the concerted activity of 12lipoxygenase and secreted phospholipase A2-IIA. Proceedings of the National Academy of Sciences of the United States of America. 2015;112(27):E3564-3573.

27. Rui-Kai Z, Jian L. Effects of xinfeng capsules on expression of platelet granule membrane protein 140 and platelet cluster of differentiation 40 ligand in peripheral blood of adjuvant arthritis rats. International journal of rheumatology. 2012;2012:139696.

28. Feng L, Zhao Y, Feng G, Chen Y. Clinical application of elevated platelet-activating factor acetylhydrolase in patients with hepatitis B. Lipids in health and disease. 2014;13:105.

29. Herrero-Beaumont G, Egido J. PAF, a potent proinflammatory mediator, looking for its role in the pathogenesis of joint damage. Annals of the rheumatic diseases. 1997;56(4):211-213.

30. Gheorghe KR, Sadique S, Leclerc P, et al. Limited effect of anti-rheumatic treatment on 15-prostaglandin dehydrogenase in rheumatoid arthritis synovial tissue. Arthritis research \& therapy. 2012;14(3):R121.

31. Pap T, Müller-Ladner U, Gay RE, Gay S. Fibroblast biology. Role of synovial fibroblasts in the pathogenesis of rheumatoid arthritis. Arthritis research. 2000;2(5):361-367.

32. Moodley D, Mody GM, Chuturgoon AA. Initiation but no execution - modulation of peripheral blood lymphocyte apoptosis in rheumatoid arthritis - a potential role for heat shock protein 70. Journal of inflammation (London, England). 2011;8(1):30.

33. Szekanecz Z, Koch AE. Mechanisms of Disease: angiogenesis in inflammatory diseases. Nature clinical practice Rheumatology. 2007;3(11):635-643.

34. Gravallese EM, Pettit AR, Lee R, et al. Angiopoietin-1 is expressed in the synovium of patients with rheumatoid arthritis and is induced by tumour necrosis factor alpha. Annals of the rheumatic diseases. 2003;62(2):100-107.

35. Schroeder M, Viezens L, Fuhrhop I, et al. Angiogenic growth factors in rheumatoid arthritis. Rheumatology international. 2013;33(2):523-527. 
36. Bhasin S, Cheung PP. The Role of Power Doppler Ultrasonography as Disease Activity Marker in Rheumatoid Arthritis. Disease markers. 2015;2015:325909.

37. D'Agostino MA, Boers M, Wakefield RJ, et al. Exploring a new ultrasound score as a clinical predictive tool in patients with rheumatoid arthritis starting abatacept: results from the APPRAISE study. RMD open. 2016;2(1):e000237.

38. Takase-Minegishi K, Horita N, Kobayashi K, et al. Diagnostic test accuracy of ultrasound for synovitis in rheumatoid arthritis: systematic review and meta-analysis. Rheumatology (Oxford, England). 2018;57(1):49-58.

39. Targońska-Stępniak B, Zwolak R, Piotrowski M, Grzechnik K, Majdan M. The Relationship between Hematological Markers of Systemic Inflammation (Neutrophil-To-Lymphocyte, Platelet-To-Lymphocyte, Lymphocyte-To-Monocyte Ratios) and Ultrasound Disease Activity Parameters in Patients with Rheumatoid Arthritis. Journal of clinical medicine. 2020;9(9).

40. Olumuyiwa-Akeredolu OO, Pretorius E. Platelet and red blood cell interactions and their role in rheumatoid arthritis. Rheumatology international. 2015;35(12):1955-1964.

41. Łukasik ZM, Makowski M, Makowska JS. From blood coagulation to innate and adaptive immunity: the role of platelets in the physiology and pathology of autoimmune disorders. Rheumatology international. 2018;38(6):959-974.

42. Naredo E, Rodríguez M, Campos C, et al. Validity, reproducibility, and responsiveness of a twelve-joint simplified power doppler ultrasonographic assessment of joint inflammation in rheumatoid arthritis. Arthritis and rheumatism. 2008;59(4):515-522.

43. El-Gohary RM, Ahmed Mahmoud AA, Khalil A, El-Gendy H, Gado KH. Validity of 7-Joint Versus Simplified 12-Joint Ultrasonography Scoring Systems in Assessment of Rheumatoid Arthritis Activity. Journal of clinical rheumatology: practical reports on rheumatic \& musculoskeletal diseases. 2019;25(6):264-271.

44. Prevoo ML, van 't Hof MA, Kuper HH, van Leeuwen MA, van de Putte LB, van Riel PL. Modified disease activity scores that include twenty-eight-joint counts. Development and validation in a prospective longitudinal study of patients with rheumatoid arthritis. Arthritis and rheumatism. 1995;38(1):44-48.

45. Ellegaard K, Torp-Pedersen S, Terslev L, Danneskiold-Samsøe B, Henriksen M, Bliddal H. Ultrasound colour Doppler measurements in a single joint as measure of disease activity in patients with rheumatoid arthritis-assessment of concurrent validity. Rheumatology (Oxford, England). 2009;48(3):254-257.

\section{Figures}

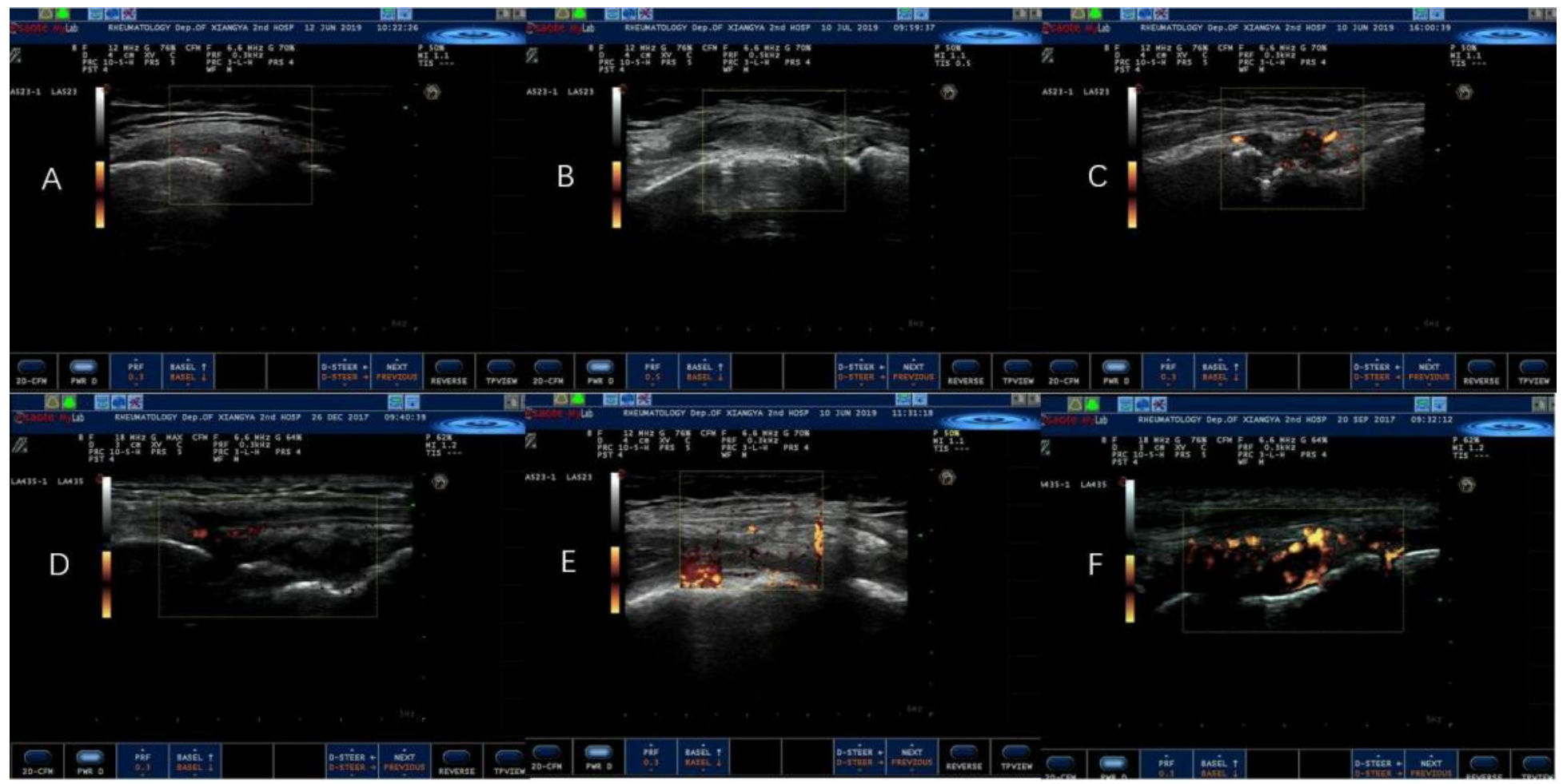

\section{Figure 1}


Representative ultrasound images of different grades of the examined joints. A: GS-US=2, PD-US=0, and T-US=2; B: GS-US=3, PD-US=0, and T-US=3; C: GS-US=2, PD-US=1, and T-US=3; $D$ : GS-US=2, PD-US=2, and T-US=4; $E$ : $G S-U S=3, P D-U S=2$, and T-US=5; F: GS-US=3, PD-

$\mathrm{US}=3$, and T-US $=6$.

Abbreviations: GS-US, grey-scale ultrasound score; PD-US, power Doppler ultrasound score; T-US, total ultrasound score.
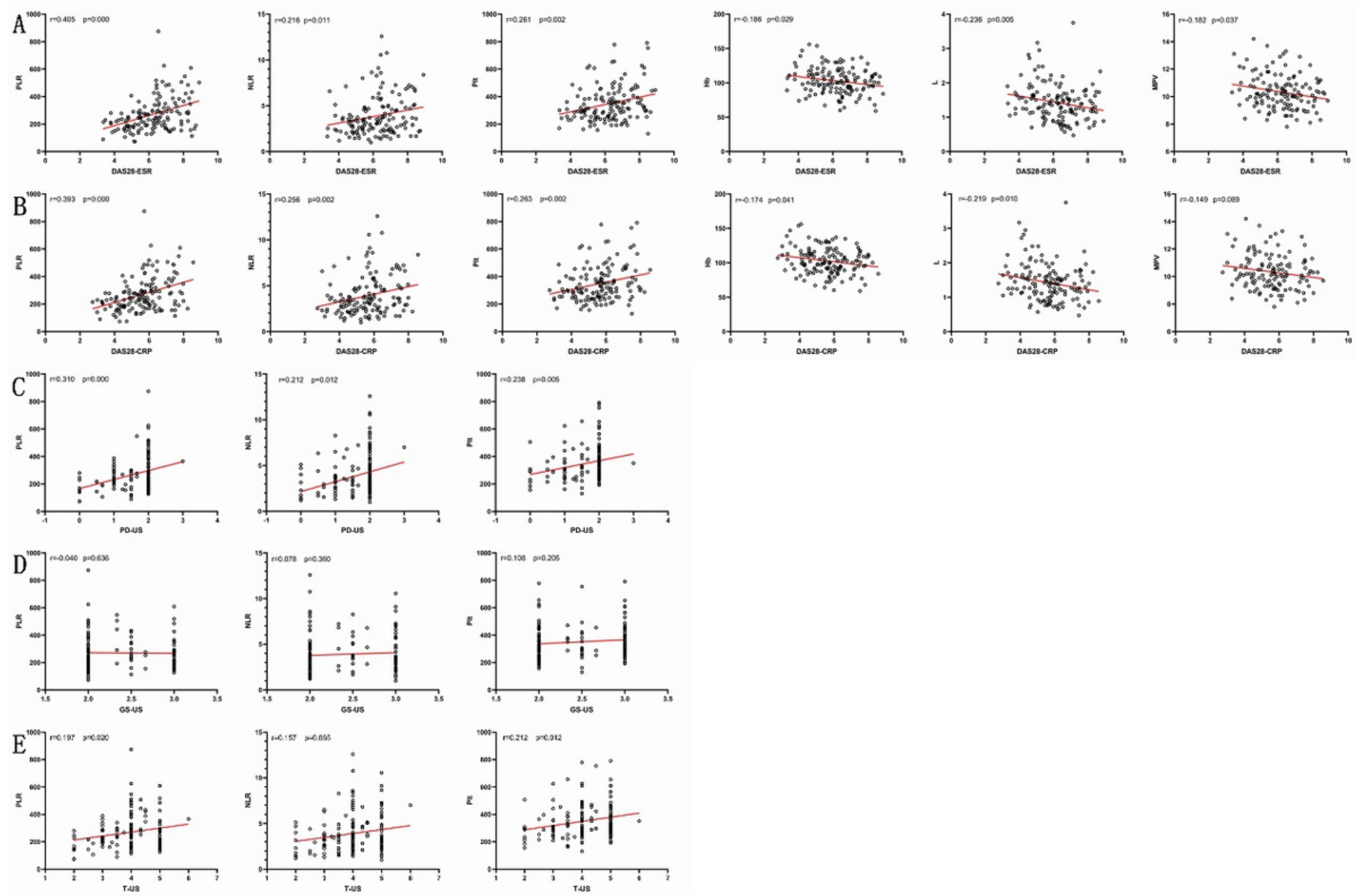

\section{Figure 2}

Association between routine hematological indices with disease activity score in 28 joints (DAS28) and ultrasound scores. A: Scatter plot of PLR, NLR, PLT, Hb, L, MPV with DAS28-ESR; B: Scatter plot of PLR, NLR, PLT, Hb, L, MPV with DAS28-CRP; C: Scatter plot of PLR, NLR, PLT with PD-US; D: Scatter plot of PLR, NLR, PLT with GS-US; E: Scatter plot of PLR, NLR, PLT with T-US.

Abbreviations: CRP, C-reactive protein; DAS28, disease activity score in 28 joints; ESR, erythrocyte sedimentation rate; GS-US, grey-scale ultrasound score; $\mathrm{Hb}$, hemoglobin; L, lymphocyte; MPV, mean platelet volume; NLR, neutrophil-to-lymphocyte ratio; PD-US, power Doppler ultrasound score; PLR, platelet-to-lymphocyte ratio; PIt, platelet; T-US, total ultrasound score. 

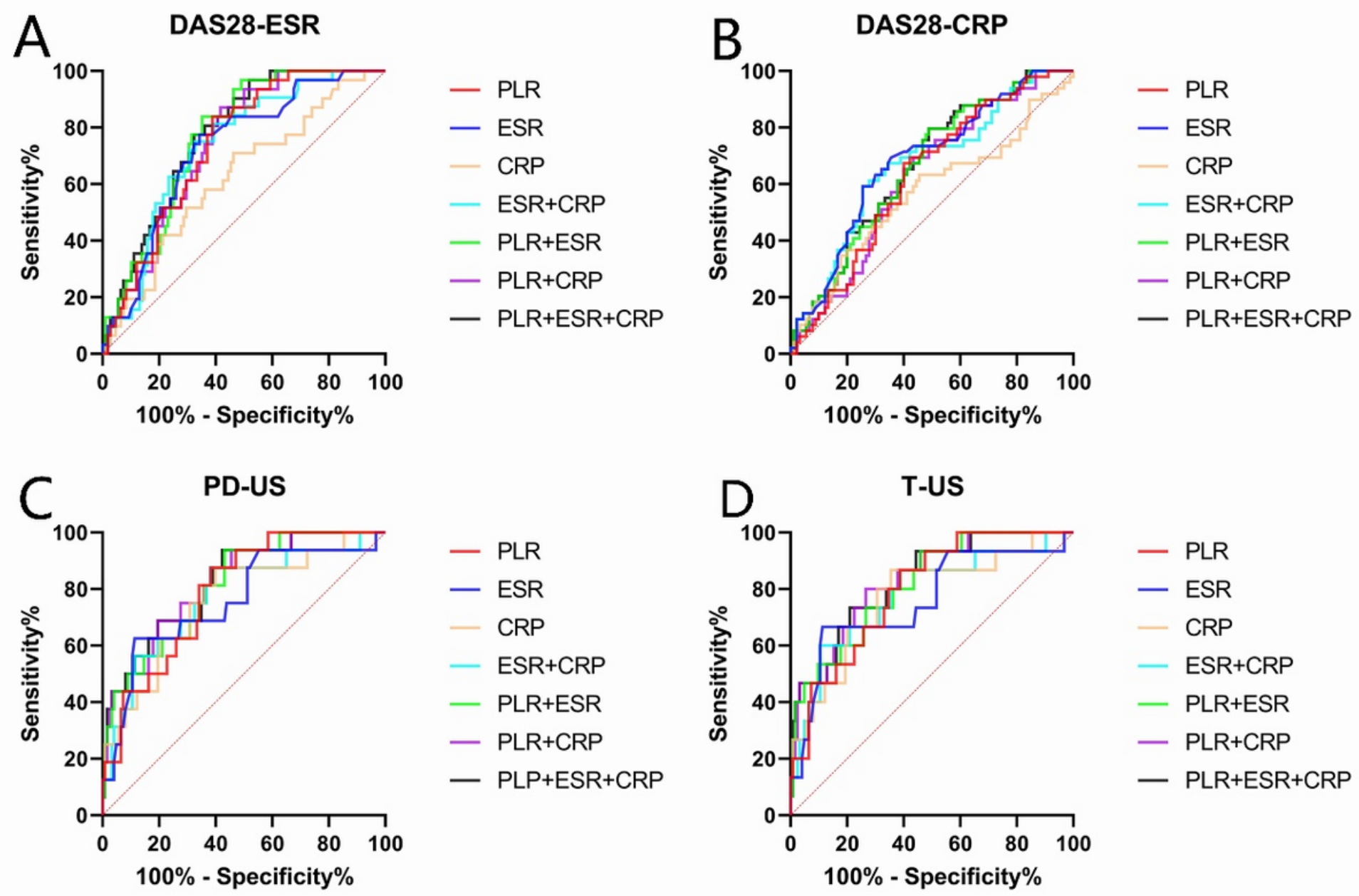

Figure 3

Receiver operating characteristic (ROC) curve analysis. A: ROC curve analysis of PLR, ESR, CRP, ESR+CRP, PLR+ESR, PLR+CRP, and PLR+ESR+CRP with DAS28-ESR as standard; B: ROC curve analysis of PLR, ESR, CRP, ESR+CRP, PLR+ESR, PLR+CRP, and PLR+ESR+CRP with DAS28-CRP as standard; C: ROC curve analysis of PLR, ESR, CRP, ESR+CRP, PLR+ESR, PLR+CRP, and PLR+ESR+CRP with PD-US as standard; D: ROC curve analysis of PLR, ESR, CRP, ESR+CRP, PLR+ESR, PLR+CRP, and PLR+ESR+CRP with T-US as standard.

Abbreviations: CRP, C-reactive protein; DAS28, disease activity score in 28 joints; ESR, erythrocyte sedimentation rate; PD-US, power Doppler ultrasound score; PLR, platelet-to-lymphocyte ratio; T-US, total ultrasound score. 